\title{
LA FRONTERA HISTORIOGRÁFICA: MÉXICO Y ESTADOS UNIDOS 1968-1988
}

\author{
Por \\ David R. Maciel y Martín González de la Vara*
}

\begin{abstract}
RESUMEN
La región fronteriza entre México y Estados Unidos ha sido objeto de numerosos estudios y publicaciones desde la perspectiva de las ciencias sociales, las humanidades y la historia. Este ensayo hace una revisión de las tendencias, interpretaciones y perspectivas de los estudios fronterizos desde finales de los sesenta hasta el presente. Las obras históricas son objeto de particular atención, aunque también se analizan otras. El ensayo concluye con algunas observaciones generales sobre el estatus actual de los estudios sobre la frontera México-Estados Unidos y ofrece sugerencias sobre áreas que requieren de futuras investigaciones.
\end{abstract}

\section{ABSTRACT}

The U.S.-Mexican border region has received extensive study and publications in the Social Sciences, Humanities and History. This essay reviews trends, interpretations and perspectives of border studies from late 1960 to the present. Historical works are stressed, although other studies are also analized. The essay concludes with general observations on the current status of U.S.-Mexican border studies and offers suggestions for areas of needed future research.

\section{INTRODUCCIÓN}

En las dos últimas décadas, la actividad historiográfica sobre México se ha impulsado y multiplicado de manera muy significativa. Nuevos estudios generales y monográficos han ahondado en muchas de las problemáticas tradicionales y otros más han abierto nuevos caminos para las investigaciones. Dentro de esta gran expansión de la historia mexicana, las fronteras - entendidas como la línea divisoria entre dos países o pueblos, las zonas aledañas a ésta y las regiones hasta donde llegan las influencias directas recíprocas de ambos países - han comenzado a recibir una alternativa muy especial por parte no sólo de historiadores, sino de otros investigadores y escritores.

- David R. Maciel. Doctor en historia latinoamericana, profesor titular de "historia latinoamericana" en la Universidad de Nuevo México en Albuquerque.

Martín González de la Vara. Maestro en historia, investigador en el Instituto de Investigaciones Históricas de la Universidad Nacional Autónoma de México. 
La atracción de las ciencias soćràles por las fronteras es muy explicable, pues la frontera es el escenario de la relación estrecha y constante de un gran número de procesos históricos y sociales que frecuentemente aparecen aislados o son menos evidentes al interior de cada país. Buena parte de los temas cruciales de las relaciones internacionales, tales como emigración, comercio, movimientos de capital, contrabando, narcotráfico, conflictos diplomáticos, etc. se manifiestan en las regiones fronterizas con una claridad y cotidianeidad que motivan su estudio formal.

\section{ANGLOAMÉRICA DE LATINOAMÉRICA Y EL PRIMER MUNDO DEL TERCERO}

El poder constatar en la realidad cómo se relacionan estas sociedades con tan dramáticas diferencias y qué clase de vínculos se establecen entre ellas ha llevado a un gran número de investigadores a interesarse vivamente en la problemática de la frontera norte de México y sur de Estados Unidos. Por la enorme multiplicidad de sus manifestaciones, la frontera ha podido ser estudiada desde los más distintos puntos de vista. En los últimos años, las investigaciones de sociólogos, politólogos, economistas y demó. grafos de ambos países han influido de forma definitiva en los estudios hechos por historiadores. Actualmente la tendencia generalizada es realizar estudios multidisciplinarios en los cuales el objeto de estudio no es estrictamente historiográfico. Por otra parte, muchos trabajos de caráctes histórico - como historias regionales, estatales, diplomáticas, etc.-tocan de manera tangencial varios de los aspectos típicamente fronterizos. De esta forma, muchas obras son consideradas como parte de la historiografía de la frontera norte de México y sur de Estados Unidos sin tener un definitivo carácter historiográfico o sin tener referencias directas con la línea fronteriza actual.

Detrás del número cada vez mayor de estudios sobre la frontera se encuentra el apoyo de diversas oficinas gubernamentales mexicanas y norteamericanas. A partir de mediados de la década de los sesenta, la peculiar evolución de la zona fronteriza llevó a los dos gobiemos a realizar nuevos programas de desarrollo y a analizar sus resultados por medio de estudios académicos. No es por ello gratuito que a finales de esa década se hayan establecido varios centros de investigación cuyo interés primordial es el estudio del desarrollo económico y social de la frontera MéxicoEstados Unidos. La primera de estas instituciones que se fundó al norte del río Bravo, fue el Border Project, auspiciado por la fundación Carnegie desde 1954 y dirigido por el antropólogo Charles L. Loomis, Años más tarde, la Universidad de California en San Diego abrió las puertas del 
Center for U. S. Mexican Studies, y, paulatinamente, se fueron estableciendo otros centros similares en las universidades de Notre Dame (U. S.Mexico Border Studies Project). Estatal de San Diego (Institute for Regional Studies of the Californias), de Texas en El Paso (Center of Inter-American and Border Studies), de Texas en Austin(Center for Mexican Studies and Migration Studies Project) y de Arizona (The Consortium of U.S. Research Program for Mexico), por mecionar algunas de las más importantes. Asimismo, en muchas otras universidades norteamericanas, especialmente las que cuentan con fuertes departamentos de estudios latinoamericanos o chicanos, se han desarrollado programas de investigación que directa $\mathrm{o}$ indirectamente se relacionan con la problemática de la frontera entre México y Estados Unidos.

En México, los programas y centros de estudios fronterizos se comenzaron a desarrollar a partir de finales de la década de los setenta. En 1979 el Colegio de México dio inicio a un programa de Estudios sobre la Frontera Norte, que a su tiempo fue el antecedente de El Colegio de la Frontera Norte (CEFNOMEX), fundado en Tijuana en 1982 y ahora extendido a otras ciudades fronterizas. Varias universidades norteñas, a su vez, han puesto en práctica programas de estudios de la frontera. Entre ellos, se encuentra, por ejemplo, el Centro de Investigaciones Históricas UNAMUABC en Tijuana, Baja California.

Con la aparición de numerosos centros de estudios fronterizos, se han multiplicado también los congresos, así como las revistas especializadas y las colecciones donde se dan a conocer y publican las investigaciones efectuadas. Entre las reuniones académicas de importacia se encuentran el Primer Simposio de Estudios Fronterizos (Monterrey, N. L., 1979), la Reunión de Universidades México-Estados Unidos sobre Asuntos Fronterizos (La Paz, B. C., 1980), el Primer Encuentro de Poetas y Narradores Jóvenes de la Frontera Norte (Cd. Juárez, Chih., 1984), el Congreso Nacional de Etnomusicología de la Frontera Norte (Saltillo, Coah., 1985), la Reunión "Border Literature-Literatura Fronteriza" (San Diego, Cal., 1986), así como varios simposios anuales que se celebran en varios de los estados fronterizos de los dos países.

Varias revistas académicas y colecciones de libros especializados en estudios fronterizos han aparecido en los dos países, destacándose por su alcance en México la Colección Frontera, auspiciada en gran medida por El Programa Cultural de las Fronteras de la Secretaría de Educación Pública, Las revistas Calafia y Estudios fronterizos (Universidad Autónoma de Baja California); Mexican Studies-Estudios Mexicanos (Universidad de California en Irvine); Aztlan. International Journal of Chicano Research (Universidad de California en Los Angeles) y Foro Internacio- 
nal (El Colegio de México) han contribuido notablemente a la publicación de estudios sobre la frontera. Otras publicaciones periódicas en las que frecuentemente aparecen articulos sobre temas fronterizos son Anglia, Arizona and the West, El grito, Historia mexicana, New Mexico Historical Review, New Scholar, Pacific Historical Review, Rural Sociology, Social Science Journal, y Southwestern Historical Quarterly.

\section{ESCUELAS HISTORIOGRÁFICAS}

Pese a que el apoyo al estudio de la frontera norte por parte de las autoridades de los dos paises es muy reciente, el origen de las investigaciones históricas sobre la frontera méxico-norteamericana datan de cuando menos un siglo. Entendemos aquí también como estudios de la frontera a aquéllos que se han hecho sobre la región norte de México o las llamadas Provincias Internas de la época colonial. Tal vez las primeras producciones historiográficas de este tipo fueron el libro de Hubert H. Bancroft, History of the Northern Mexican States and Texas (San Francisco, The History Company, 1988) y otras obras suyas dedicadas a los estados fronterizos norteamericanos. Treinta años más tarde, Herbert Eugene Bolton escribio un primer ensayo de síntesis de la historia colonial de la región fronteriza en The Spanish Borderlands: A Chronicle of Old Florida and the Southwest (New Haven, Yale University Press, 1921). Este libro dio origen a una escuela historiografica norteamericana - conocida como The Spanish Borderlands - que se dedicó exclusivamente al estudio de la historia colonial de las regiones nortenlas de la Nueva Espania.

Durante medio siglo, la orientación de esta escuela dominó el carácter de los estudios históricos sobre el llamado Septentrión, dando lugar a la publicación de numerosas obras monográficas en ese periodo. En 1970, John Francis Bannon intento reformular una nueva síntesis dentro de los cánones de esta escuela y publico The Spanish Borderlands Frontier, 1531-1821 (New York, Holt, Rinehart and Winston, 1970) con la intención de cerrar un ciclo iniciado con los trabajos pioneros de Bolton. Esta tendencia historiográfica, lejos de su aquilosamiento, está todavía vigente en muchos centros de investigación de los Estados Unidos, produce innumerables trabajos y ha abierto sensiblemente sus horizontes de investigación. De los trillados temas iniciales referentes a la exploración del norte novohispano, la colonización "española", la evangelización de los indígenas y la organización militar se ha pasado al estudio de la demografía, las estructuras económicas y sociales y las manifestaciones culturales. Con estos nuevos objetos de investigación, las obras de historiadores como Felix D. Almaraz Jr., Henry F. Dobyn, Oddie Faulk, Mario Góngora, 
Ramón Gutiérrez, Elizabeth H. A. Johnson, Oakah L. Jones, John L. Kessell, Michael Mathes, Max L. Moorhead, David J. Weber, y muchos más, han logrado revitalizar a esta tradicional escuela.

La lógica contraparte norteamericana de la escuela de The Spanish Borderlands es aquélla que se dedica al análisis del movimiento expansionista, la colonización de los Estados Unidos hacia el oeste, es decir, al estudio de la frontera en el concepto a que Frederick Jackson Turner forjó en 1893. Debido a su peculiar orientación etnocentrista, esta escuela -conocida como Western Americana o Westward Movement-frecuentemente desdeña el estudio de los fenómenos de relación entre las sociedades mexicana y norteamericana para dedicarse al estudio de esta última. Pese a que esta escuela se muestra renuente a aceptar cambios en las formas y temas de investigación, los seguidores de Walter Prescott Webb, Paul Horgan y Ray Allen Billington son muy numerosos, en especial en las universidades del centro y lejano oeste de los Estados Unidos.

En México, el estudio científico de estos temas coloniales se puede considerar como iniciado con los trabajos de Vito Aleccio Robles, especialmente con su Coahuila y Texas en la época colonial (México, Cultura, 1938), pero su desarrollo ulterior ha sido más bien modesto, si se le compara a la producción historiográfica producida al norte del río Bravo. Es hasta las últimas dos décadas cuando los historiadores profesionales empiezan a sobrepasar en número a los cronistas regionales como productores de obras históricas sobre las Provincias Internas de la Nueva Espafia. Afortunadamente, ahora son más comunes los estudios académicos de personas con formación universitaria como Israel Cavazos, Miguel León Portilla, José Luis Mirafuentes, David Piñera, Guillermo Porras Mufioz, Ignacio del Río, María del Carmen Velázquez o Isidro Vizcaya Canales y se vuelve común el hecho de que en las universidades estataies del norte de México existan institutos de investigaciones históricas.

La historia de los estados y regiones fronterizas se ha desarrollado también desde hace aproximadamente un siglo y ha estudiado con frecuencia, aunque la mayor parte de las veces de manera tangencial, los problemas inherentes a la frontera mexicano-norteamericana. En Estados Unidos a este tipo de historiografía se le ha llamado tradicionalmente United States Southwest History y versa sobre las historias de los estados de Arizona, California, Colorado, Nuevo México y Texas, así como sus subregiones y localidades. En México, mientras tanto, este tipo de historiografía es conocida generalmente como historia o microhistoria. En ambos países, ha sido notable la profesionalización de esta clase de estudios regionales en los últimos años y su número creciente de publicaciones. Hoy en día se están realizando abundantes investigaciones de estudiosos como Francisco 
Almada, Mario Cerruti, Daniel Tyler, Clark S. Knowlton, Oscar J. Martínez, Angela Moyano, Gerlad Nash y David Pifiera, Marc Simmons, por mencionar sólo algunos, que nos dan una idea más completa sobre la participación de las regiones fronterizas de los dos paises en los procesos que conforman sus respectivas historias nacionales.

Los complejos fenómenos que se han desarrollado en la frontera entre México y Estados Unidos en el siglo XX, por su misma magnitud, han sido estudiados por una notable cantidad de científicos sociales, predominando en los llamados estudios fronterizos - o Border Studies - una visión multidisciplinaria de los objetos de estudio. Este tipo de estudios ha experimentado un enorme crecimiento en las pasadas dos décadas y también su influencia ha sido cada vez mayor en las políticas fronterizas de los dos países. Desde los trabajos seminales de Manuel Gamio y Paul $S$. Taylor, realizados a finales de la década de los veinte, hasta la actualidad el número de instituciones, investigaciones y publicaciones sobre temas de la frontera ha aumentado a un ritmo tan veloz que es díficil seguir la pista del progreso de los estudios fronterizos en las distintas ciencias sociales y humanidades.

A finales de la década de los sesenta, cuando en los Estados Unidos el llamado movimiento chicano entraba a su apogeo, surgió una importante escuela historiográfica que también contempla con frecuencia la problemática de la frontera: la historia chicana. Con un explosivo desarrollo en menos de veinte años, los investigadores chicanos han aportado valiosos datos y perspectivas a la historia de la frontera entre México y Estados Unidos gracias a sus trabajos bien documentados y a su visión innovadora y crítica de la realidad social de ambos países. Estudios como Occupied America, The Chicano's Struggle Toward Liberation (New York, Harper and Row, 1970) de Rudolfo Acuña; Race and Class in the Southwest (Notre Dame, University of Notre Dame, 1979) de Mario Barrera; Dessert Immigrants, the Mexicans at El Paso (New Haven, Yale University Press, 1971) de Mario T. García; Anglos and Mexicans in the Making of Texas (Austin, University of Texas at Austin, 1987) de David Montejano, y otros trabajos de Alberto Carrillo, Pedro Castillo, Tobías Durán, Juan GómezQuiñones, Richard Griswold del Castillo, Gilberto Hinojosa, Annoldo De León, David R. Maciel, Ricardo Romo, Renato Rosaldo, Robert J. Rosenbaum, Vicki Ruiz, Emilio Zamora y muchos más contienen material y análisis importantes sobre la frontera México-Estados Unidos.

La producción histórica acerca de la frontera norte de México y sur de Estados Unidos es, sin embargo, de tan variadas características que en muchas ocasiones rebasan los estrechos límites de las escuelas historiográficas antes descritas al abordar los diversos temas de la vida fronteriza 
desde otros puntos de vista, como, por ejemplo, de la historia de las relaciones diplomáticas o las historias económica, demográfica, militar y cultural.

\section{ESTUDIOS GENERALES Y BIBLIOGRÁFICOS}

Un factor que ha contribuido a la publicación de un número cada vez creciente de estudios sobre la región fronteriza entre México y Estados Unidos es el lanzamiento público de algunas colecciones bibliográficas especializadas en los estudios fronterizos. En las instituciones que han echado a andar recientemente programas de investigación sobre el tema se han establecido series de cuademos de trabajo como la Border Studies Series o la Border Issues Series de dos universidades de California. De impacto semejante es la Serie Frontera auspiciada por la Secretaría de Educación Pública a través del Programa Cultural de las Fronteras. Hasta la fecha se han publicado una docena de volúmenes, varios de los cuales - Las aventuras de don Chipote o cuando los pericos mamen, El movimiento obrero mexicano en el sur de Texas, y México-Estados Unidos: origenes de una relación, 1819-1861 - tocan de lleno temas históricos de la frontera norte de México. Los libros que anualmente publica El Centro de Estudios Internacionales de El Colegio de México con el título México-Estados Unidos, son también útiles para seguir los últimos ensayos sobre las relaciones entre ambos países, muchos de los cuales tratan acerca de la problemática contemporánea de la frontera mexicano-norteamericana.

La historia de la frontera mexicano-norteamericana ha sido abordada de manera general desde la perspectiva de varias ciencias sociales afines a la historiografía. Por los actuales requerimientos de las políticas nacionales, tiende a predominar en los estudios generales el enfoque económico-administrativo sobre el historiográfico propiamente dicho, de forma que es notable en este tipo de obras su estilo descriptivo, la carencia del uso de fuentes primarias y la poca originalidad en las hipótesis de trabajo. Destaca por su seriedad y amplia visión el estudio de Raúl A. Femández: The U. S.-Mexico Border, A Political-Economic Profile (Notre Dame, Indiana, University of Notre Dame, 1977) que contiene una descripción panorámica de los procesos económicos más importantes que han moldeado la vida de la frontera en los siglos XIX y XX. Otros libros generales que dan una gran importancia al aspecto económico han sido el resultado de reuniones académicas sobre la frontera. Por ser obras colectivas e incluir, por lo general, a especialistas mexicanos y estadounidenses, integran y hacen contrastar sus diversas opiniones, por lo que no pueden considerarse, strictu sensu, como obras de síntesis. En este caso se encuentran: Views 
Across the Border. The United States and Mexico (compilada por Stanley R. Ross, Albuquerque, University of New Mexico Press, 1979), La frontera norte; integración y desarrollo (editada por Roque González Salazar, México, El Colegio de México, 1981), Estudios fronterizos, Ecología y desarrollo en la región fronteriza y Reglas del juego y juego sin reglas en la vida fronteriza (publicados por la ANUIES en 1981). Mientras tanto, el libro de Mario Ojeda Administración del desarrollo de la frontera norte (México, El Colegio de México, 1987) trata más de cerca el desarrollo económico de la frontera norte en este siglo $\mathrm{XX}$.

La geografía histórica, más apropiada por su objeto de trabajo para brindar una visión panorámica de la historia fronteriza, ha aportado algunos trabajos de sumo interés a los estudios fronterizos. El manual de Peter Gerhardt, The Northern Frontier of New Spain (Princeton, New Jersey, Princeton University Press, 1977) forma parte de una serie de tres libros dedicados a la geografía de la Nueva España en la que el autor busca principalmente dilucidar las jurisdicciones administrativas novohispanas. Por la seriedad de la investigación de fuentes primarias es un instrumento invaluable para los estudios de las Provincias Internas. Otros estudios sobre el tema son: Southwest: Three Peoples in Geographical Change. 1600-1970, de D. W. Meinig (New York, Oxford University Press, 1971); Los chicanos: geografta histórica regional, de Richard L. Nostrand (Mé.xico, Secretaría de Educación Pública, 1972); El noroeste de México: un estudio geográfico y económico, de Angel Bassols Batalla (México, Instituto de Investigaciones Económicas, UNAM, 1972); Frontier in the Rio Grande. A Political Geography of Development and Social Deprivation. de John House (New York, Oxford University Press, 1982); asi como varios artículos de Charles R. Gildersleeve y ClarckL. Knowlton muestran el desarrollo de la colonización de las regiones fronterizas de los dos países en distintas épocas.

El estudio de las vicisitudes y problemas para la delimitación de la línea fronteriza en sí ha sido uno de los temas recurrentes de la historiografía diplomática tanto en Estados Unidos como en México desde hace muchas décadas. En años recientes, la apertura de nuevos temas de investigación ha llevado a la historia diplomática a un relativo abandono; sin embargo, se ha producido alguna obra sobre estos temas como La frontera norte de México. Historia. Conflictos. 1762-1975, de César Sepúlveda (México, Porrúa, 1976).

Otro tipo de estudios generales de la frontera está enfocado al estudio de la vida social en las regiones fronterizas. Algunos libros como The Border, Life on the Line (New York, Abbeville, 1988) de Douglas Kent; La Frontera; The United States Border with Mexico, de Allan Weisman y 
Jay Dusand y On the Border; Portraits of America's Southwestern Frontier (New York, Harper and Row, 1981) de Tom Miller, sirven de introducción general al tema a partir de sus excelentes fotografías.

Pese a la gran producción de libros de temas fronterizos, los estudios generales estrictamente historiográficos son tadavía escasos. Destaca como primeros intentos de síntesis la trivoluminosa obra colectiva Visión historica de la frontera norte de México (México, Centro de Investigaciones Históricas UNAM-UABC, 1987). Este libro, integrado por sesenta y dos colaboraciones de distinta factura por alrededor de setenta especialistas en lu historia del norte mexicano, la mayor parte de ellos mexicanos, es hasta uhora la mejor síntesis de la historia de los estados norteños de México y una obra de consulta obligada sobre el tema. Por otra parte, el trabajo de Romeo Flores Caballero: La frontera entre México y Estados Unidos. Desarrollo histórico (Monterrey, Facultad de Ciencias Sociales, UANL, 1976 y reeditado por El Centro de Investigaciones Económicas de la UANL on 1982 con el título Evolución de la frontera norte) muestra poca originalidad y escaso manejo de fuentes primarias, aunque se le considera una obra de consulta aceptable.

$\mathrm{La}$ inmensa producción de estudios fronterizos en las distintas ramas do las ciencias sociales dio lugar a que, cuando menos desde 1960, se comenzaran a elaborar bibliografías sobre el tema. En ese año, Charles C. Cumberland publicó en la revista Rural Sociology (vol, XXv, no. 2, junio do 1960) "The United States- Mexico Border: A Selective Guide to the Litcrature to the Region", que es el primer esfuerzo de reunir y ordenar los ostudios fronterizos existentes hasta esa época. En los años que siguieron, Iu producción bibliográfica ha aumentado de forma considerable $\mathrm{y}$, de ucucrdo con este crecimiento, se han hecho nuevas biliografías y ensayos do interpretación sobre los avances de diversas disciplinas en cuanto al owtudio de temas fronterizos. En 1975, el Social Science Journal (vol. XII, no. 3, octubre de 1975) dedicó un número especial para los estudios Ironterizos - titulado "The Status of Borderlands Studies" - en el cual vurios investigadores norteamericanos evaluaron los avances que la antropologfa, historia, sociología, ciencia política, geografía, economía y los oxtudios literarios habían hecho hasta entonces en relación con los fenómunos fronterizos. Casi una década más tarde, New Scholar (no. 9, 1984) publicó otra serie de ensayos con el mismo objetivo. Estas dos publicacionow especiales siguen siendo fundamentales para el estudio de la frontera, nith embargo, ya se han visto superadas por nuevas obras de referencia.

El libro México-Estado Unidos. Bibliografia general de estudios fronicrizos (México, El Colegio de México, 1981), preparado por Jorge A. Ilustamante y Francisco Malagamba es recomendable para los estudiosos 
de la economía, aunque desdefia un poco los temas estrictamente historiográficos. De indudable mayor utilidad para los historiadores es, por ejemplo, el libro de Ellwyn R. Stoddard, et al, (coords.) Borderlands Sourcebook. A Guide to the Literature on Northern Mexico and the American Southwest (Norman, University of Oklahoma Press, 1983), que contiene un reporte completo del avance de varias ramas sociales e incluye un resumen sobre el desarrollo historiográfico de temas fronterizos, además de una amplísima bibliografía. El trabajo colectivo Mil tres textos sobre la historia de la frontera norte (México, Comité Mexicano de Ciencias Históricas, 1986), aunque presenta una calidad irregular en el análisis de la historiografía de cada estado norteño de México, tiene el valor de rescatar muchos títulos de la historia regional y complementar el cuadro bibliográfico a los ensayos elaborados al norte del río Bravo, los cuales tienden a dejar fuera la producción historiográfica mexicana.

Varios autores, entre ellos Luis Leobaldo Arroyo, Albar W. Carlson, Arthur F. Corwin, Juan Gómez-Quiñones, Luis G. Nogales, y Lyle Saunders, han escrito numerosas bibliografias relacionadas con la historia chicana, tantas, que resulta más útil consultar directamente la "Bibliography of Bibliographies Relating to Mexican Studies" de Joseph A. Clark y Moreno (El Grito, vol. III, Verano de 1970) o Mexican American Bibliographies, de Carlos E. Cortés (New York, Amo Press, 1974). Existen de igual manera abundantes bibliografias relacionadas con estudios económicos y demográficos de la frontera escritas por John D. Buenker, Jorge Carrillo, Beverly Fodell, Manuel García y Griego, Matt S. Meier, Feliciano Rivera, Nancy Saldaña y muchos otros que contienen citas de obras históricas.

\section{ESTUDIOS MONOGRÁFICOS}

La gran mayoría de los estudios fronterizos pueden clasificarse como monográficos, una minoría de los trabajos es de sintesis general y casi inexistentes las obras que se encuentran a medio camino entre estos dos extremos. La diversidad de temas relacionados con la frontera norte de México y sur de Estados Unidos obliga entonces a un examen, aunque breve, de la producción historiográfica acerca de cada periodo de su desarrollo.

\section{a) Época colonial}

Los casi trescientos años de historia que abarcan desde las primeras exploraciones de los pioneros españoles en tierras chichimecas hasta la 
consumación de la independencia de la Nueva España en 1821 constituyen, dentro de la historia de la frontera, uno de los periodos mejor estudiados desde finales del siglo pasado. Desde la constitución de la escuela norteamericana conocida como The Spanish Borderlands, en la década 1920-1930 hasta nuestros días, se han producido incontables trabajos en ambas partes de la frontera, elaborados casi exclusivamente por historiadores, sobre los distintos aspectos que constituian a la sociedad novohispana establecida fuera de los límites de Mesoamérica. Muchas de estas investigaciones nuevas se siguen dando a conocer a través de revistas especializadas de una gran tradición como Arizona and the West, The Americas, Estudios de historia novohispana, Hispanic American Historical Review, Historia mexicana, Journal of the West, New Mexico Historical Review, Southwestern Historical Quarterly, Texana, y muchas más.

Pese a la gran cantidad de monografías que existen sobre estos temas, los estudios generales sobre la frontera norte de la Nueva Espana son relativamente escasos. Las antologias de Oakah L. Jones, The Spanish Borderland: A First Reader (Los Angeles, Lollin I. Morrison, 1974) y de David J. Weber, El México perdido: Ensayos sobre el antiguo norte de México, 1540-1821 (México, Secretaría de Educación Pública, 1976) son una excelente introducción al tema a través de los ensayos más significativos que se publicaron desde principios de este siglo, en los cuales se muestran con claridad las diversas posturas de los autores más reconocidos e influyentes sobre temas fronterizos. Entre las síntesis, la obra ya citada de John Francis Bannon, The Spanish Borderlands Frontier, 1512-1820, hace una excelente sinopsis de los temas tradicionales de investigación de la escuela fundada por su maestro Bolton; es decir, la exploración, colonización y las actividades políticas y militares. Los Paisanos; Spanish Settlers on the Northern Frontier of New Spain (Norman, University of Oklahoma Press, 1979) de Oakah L, Jones, en cambio, realiza con buen éxito el análisis y descripción de las estructuras económicas, sociales y culturales que integraban una sociedad fronteriza conformada, más que por las autoridades, por los mismos pobladores de las Provincias Internas.

Las grandes proezas de la exploración, evangelización indígena, conquista y colonización de amplísimos territorios, en un periodo de tiempo relativamente corto, despertó desde un principio la curiosidad de los investigadores, por lo que el grueso de las investigaciones sobre estos temas se han producido en los años anteriores a 1970. Estos temas tradicionales de investigación, sin embargo, siguen motivando la aparición de numerosas monografías que han aportado nuevos datos, hipótesis e interpretaciones que tienden a mostrar las diferencias regionales con que estos procesos coloniales se fueron desarrollando en las distintas provin- 
cias que conforman el norte novohispano. La historia colonial de las Californias ha sido una de las más estudiadas por investigadores mexicanos y norteamericanos desde hace varias décadas, sin embargo, aún existe un gran interés principalmente en el estudio de su exploración y el establecimiento de sus misiones. Travelers among the Cucupa (Los Angeles, Dawson's Book Shop, 1975) de Anita Álvarez de Williams; Jesuit Relations. Baja California 1719-1762 (Los Angeles, Dawson's Book Shop, 1984); Los jesuitas californianos (México, s. e., 1975) de Francisco Javier Carballo; The King's Highway in Baja California: An Adventure into the History and Lore of a Forgotten Region (San Diego, Copley Books, 1974); Franciscan Missionaries in Hispanic California, 1769. 1848 (San Marino, Huntington Library, 1969) de Maynard J. Geiger; De México a la Alta California; Una gran epopeya misional (México, Jus, 1969) de Lino Gómez Cahedo; Las misiones de Baja California, J6831849 (La Paz, Ayuntamiento de La Paz, 1977), Sebastián Vizcalno y la expansión española en el océano Paclfico, 1580-1630 (México, Instituto de Investigaciones Históricas, UNAM, 1973) y la gran recopilación documental Californiana (Madrid, Porrúa Turanzas, 1974) de Michael Mathes; $A$ la diestra mano de las Indias. Descubrimiento y ocupación de la Baja California (La Paz, Gobierno del Estado, 1985) y Conquista y aculturación en la California Jesuítica, 1697-1768 (México, Instituto de Investigaciones Históricas, UNAM, 1984) de Ignacio del Río son sólo algunos de los libros más importantes que forman parte de una abultada bibliografía publicada sobre temas californianos en los últimos años.

La región de Sonora y Arizona, que había sido un poco relegada en las primeras décadas de este siglo con excepción de varios trabajos dedicados a las labores misionales del padre Kino, cuenta ahora con estudios de gran calidad como: Kino and Manje, Explorers of Sonora and Arizona, Their Vision of the Future: A Study of Their Expeditions and Plans (Roma, Jesuit History Institute, 1971) de Ernest J. Burrus; After Kino: Jesuit Missions in Northwestern New Spain, 1711-1767 (Roma, Jesuit History Institute, 1969) de John A. Donohue; Etnologla y misión en la Pimería Alta (1715-1740) (México, Instituto de Investigaciones Históricas, UNAM, 1977) de Luis González Rodríguez; Missionaries, Miners and Indians: Spanish Contact with the Yaqui. Nation of Norlhwestern New Spain, 1533-1820 (Tucson, University of Arizona, 1981) de Evelyn Hu de Hart; Missions of Sorrows, Jesuit Guevavi and the Pimas, y Friars, Soldiers and Reformers. Hispanic Arizona and the Sonora Mission Frontier, 17671856) (Tucson, University of Arizona, 1970 y 1976 respectivamente) de John L. Kessell; A Spanish Frontier in the Enlightened Age, Franciscan Beginnings in Sonora and Arizona, 1767-1770 (Washington, Academy of 
Amorican Franciscan History, 1981) de Kieran R. Mc Carty; Rules and Irecepts of the Jesuit Missions of Northwestern New Spain (Tucson, Unlversity of Arizona, 1976) de Charles W. Polzer; y Las estructuras nocloeconomicas de las misiones de la Pimerla Alta, 1768-1850 (Hermoillo, Centro Regional del INAH, 1979) de Cynthia Radding de Murrieta.

Las regiones centrales de la frontera norte novohispana han recibido unu atención muy dispar en sus historias regionales, pues mientras que nobre Nuevo México se encuentran abundantes estudios históricos desde fechas muy tempranas, Chihuahua carece casi por completo de trabajos nerios acerca de los más importantes aspectos de su desarrollo colonial. El impresionante número de publicaciones que sobre la historia de la conquista y evangelización de Nuevo México se escribieron entre 1880 y 1968 ha hecho muy difícil la aparición de nuevos filones de investigación, de manera que en las últimas décadas poco se ha podido avanzar en estos temas tan trillados. Sin embargo, algunos estudios que se integran más a la antropología que a la historiografía propiamente dicha, han podido arrojar nuevas luces sobre el sistema misional nuevomexicano: The Jicarilla Apache Tribe; Historical Documents (New York, Gerland Publications, 1974) de Donald C. Cutter; Kiva, Cross ans Crown: The Pecos Indians and New Mexico, 1540-1840 (Washington, National Park Service, 1979) y The Missions of New Mexico since 1776 (Albuquerque, University of New Mexico, 1980) de John L. Kessell; The Apache Frontier, Jacobo de Ugarte and Spanish-Indian Relations in Northern New Spain, 1769. $179 i$ (Norman, University of Oklahoma, 1968) de Max L. Moorhead; Cycles of Conquest: The Impact of Spain, Mexico and the United States on the Indians of the Southwest, 1533-1960 (Tucson, University of Arizona, 1972) de Edward H. Spicer; y The Conquest of the Apacheria (Norman, University of Oklahoma, 1968) de Dan L. Thrapp.

Los temas coloniales chihuahuenses, mientras tanto, apenas ahora comienzan a ser tratados en forma científica por historiadores profesionales. Las obras de Guillermo Porras Muñoz, Iglesia y Estado en Nueva Vizcaya (1562-1821) (México, Instituto de Investigaciones Jurídicas, 1980) y La frontera con los indios de la Nueva Vizcaya en el siglo XVII (México, Fomento Cultural BANAMEX, 1980), y la compilación de Lino Gómez Canedo Las misiones de Nueva Vizcaya en el siglo XVIII. Los afanes secularizados del obispo Tamarón (Guadalajara, Universidad Autónoma de Guadalajara, 1977), parte de la escasa bibliografía reciente sobre temas novovizcaínos, muestra gran interés en la colonización de la Nueva Vizcaya y la misiones en la región tarahumara.

La frontera nororiental de la Nueva España, en cambio, ha motivado un buen número de investigaciones en los últimos años; sin embargo, por 
ser estas las provincias norteñas de más tardía colonización y menor actividad misional, los temas de la colonización y conquista no han sido tan favorecidos por los estudiosos como algunos otros aspectos de su vida colonial, De todas formas, han salido recientemente a la luz algunas obras que tocan estos temas como: Spanish Texas (Austin, Pemberton Press, 1971) de Gerald Ashford; Primeras exploraciones y poblamiento de Texas (1686-1694) (Monterrey, ITESM, 1968) de Lino Gómez Canedo; Las misiones franciscanas en la colonia del Nuevo Santander (Ciudad Victoria, Instituto de Investigaciones Históricas, UAT, 1975) de Carlos González Salas; The Alamo Chain of Missions: A History of San Antonio's Five Missions (Chicago, Franciscan Herald Press, 1968) de Marion A. Habing; El carácter de la conquista y la colonización española en el noreste de México, siglo XVI y siglo XVII (Monterrey, ITESM, s. f.), Historia del Nuevo Reino de León (1577-1723) (Monterrey, ITESM, 1972) e Indios, frailes y encomenderos en el Nuevo Reino de León, siglos XVI y XVII (Monterrey, AGENL, 1985) de Eugenio del Hoyo; y San Juan Bautista; Getaway to the Spanish Texas (Austin, University of Texas, 1968) de Robert S. Weddle.

Los aspectos institucionales y político-administrativos han sido una veta muy explotada de los estudios coloniales fronterizos desde la aparición de la obra clásica de William Blackmar Spanish Institutions in the Southwest (Baltimore, John Hopkins University, 1981). A mediados de la década de los cuarenta, Frances V. Scholes dio un paso definitivo, con sus trabajos sobre Nuevo México, para popularizar esta temática entre los investigadores. Sin duda alguna, la institución que más estudios académicos se ha merecido es el presidio, sobre el cual se ha desarrollado un amplio debate acerca de sus funciones y eficacia como motor de la colonización del norte novohispano. A principios de 1969, el especialista en la historia colonial de Texas y autor de varios libros y ensayos publicó un artículo titulado "The Presidio, Fortress or Farce?" (Journal of the West, vol. VIII, no. 1, enero de 1969) en el cual expuso con crudeza los aspectos negativos del sistema presidial, desmitificando su significado y simbolismo como institución fronteriza. En ese mismo número Max L. Moorhead exponía la tesis contraria en "The Soldado de Cuera: Stalwart of the Borderlands", Estos ensayos produjeron una fuerte reacción entre los historiadores que llevó a la publicación de varios artículos y libros de gran valor sobre el tema en los siguientes años, en los que se juzga el papel jugado por el presidio en el norte de la Nueva España. Defendiendo la tesis del colapso del sistema defensivo fronterizo, Faulk publicó posteriormente The Leather Jacket Soldier: Spanish Military Equipment and Institutions of the Late 18th Century (Pasadena, Socio-Technical Publications, 1971), y otros autores, como Richard W. Gronet y Paige W. Christiansen, lo apoyaron 
ton diversos libros y artículos. Favoreciendo a esta institución se escribieron: The Spanish Royal Corps of Engineers in the Western Borderlands: Instrument of Bourbon Reform, 1764-1815 (Glendale, Arthur H. Clark, 1977) de Janet Fireman; The Presidio, Bastion of the Spanish Borderlands (Norman, University of Oklahoma, 1975) de Max L. Moorhead; La frontera norle y la experiencia colonial (México, Secretaría de Relaciones Exteriores, 1982) de María del Carmen Velázquez y algunos artículos de Myra Ellen Jenkins, Ted J. Warner, Christon Archer y William A. DePalo.

El estudio de las estructuras político-administrativas ha sido un tema que sigue atrayendo a los historiadores, aunque con menos fuerza con que lo hacian a mediados de este siglo. Sobre estas cuestiones ha salido a la luz, entre otras investigaciones: El muy ilustre Ayuntamiento de Monterrey desde 1596 (Monterrey, 1980) y Controversias sobre jurisdicción espiritual entre Saltillo y Monterrey (Saltillo, Instituto Coahuilense de Investigaciones Históricas, 1973) de Israel Cavazos Garza; Las Provincias Internas en el siglo XIX (Sevilla, Escuela de Estudios Hispanoamericanistas, 1968) de Luis Navarro García; Spanish Government of New Mexico (Albuquerque, University of New Mexico, 1968) de Marc Simmons; y El poder colonial en el Nuevo Santander (México, Porrúa, 1976) de Juan Fidel Zorrilla. La historia política de la provincia de Texas muestra la particularidad de su participación en la guerra de Independencia, y sobre todo este aspecto se han escrito Tragic Cavalier: Governor Manuel Salcedo of Texas, 1808-1813 (Austin, University of Texas, 1971) de Felix D. Almaraz; y De los albores de la Independencia, Las Provincias Internas de Oriente durante la insurrección de don Miguel Hidalgo y Costilla, 1810-11 (Monterrey, ITESM, 1976) de Isidro Vizcaya Canales.

La economía y la sociedad de la frontera norte de la Nueva España se ha constituido como uno de los temas de investigación relativamente más recientes, que se ha comenzado a desarrollar a partir de las monografías de Peter J. Bakewell y David Brading sobre la miseria y el comercio en otras zonas de la Nueva España. Casi todos estos trabajos se refieren a la economía agrícola, y entre ellos se puede incluir a La Nueva Villa de Croix y el repartimiento de sus tierras (Ciudad Victoria, s. e., 1977) de Vidal Efrén Covián Martínez; A Mexican Family Empire; The Latifundio of the Sanchez Navarros (Austin, University of Texas, 1975) de Charles H. Harris; The Ranch in Spanish Texas (El Paso, Texas Western Press, 1979) de Sandra L. Myres; y Royal Land Grants North of the Rio Grande, 1777-1821 (s.1., Texian Press, 1969) de Florence J. Scott. 


\section{b) Siglo XIX}

En el siglo pasado se estableció la frontera entre México y los Estados Unidos tal cual la conocemos. Es entonces lógico que los temas historiográficos de este periodo respondan por lo general a la temática de los primeros encuentros y conflictos que se dieron en la temprana relación entre estos dos países. Es indudable que los investigadores le han prestado una menor atención a la historia de la frontera mexicano-norteamericana en el siglo XIX que a la de los tiempos coloniales, aun así, se puede contar con algunos trabajos de síntesis que se salen de la tendencia generalizada a tratar este periodo dentro de las distintas historias estatales. Los libros Land of Many Frontier: A History of the American Southwest (New York, Oxford University, 1968) de Odie Faulk; The American Southwest, Its Peoples and Cultures (New York, Holt, Rinehart and Winston, 1971) de Lynn I. Perrigo y The Southwest, (Albuquerque, University of New Mexico, 1980) de David Lavender, aunque tratan la historia de esa región desde los tiempos coloniales hasta los contemporáneos, nos dan una síntesis muy general de los procesos que desencadenaron en la guerra de 1846-1848 y la pérdida de esos territorios por parte de México. De mayor utilidad es el libro de David J. Weber The Mexican Frontier, 1821-1846; The American Southwest underMexico (Albuquerque, University of New Mexico, 1982). Con una amplia bibliografía, material de primera mano y un buen manejo de las fuentes, Weber intenta mostrar la perspectiva mexicana a través de los problemas del gobierno central para conservar su soberanía sobre esa amplia región. Según el autor, las provincias norteñas, carentes casi por completo de representación política, testigos de la decadencia de las instituciones coloniales como el presidio y las misiones, y dominadas económicamente por el creciente influjo del capital norteamericano ante la impotencia del gobierno de México, desarrollaron una gran dependencia económica y cultural de los Estados Unidos que culminó lógicamente con la agregación de esos territorios a este país. De ahí la contradicción reflejada en el subtítulo El suroeste norteamericano bajo México. Hay que hacer notar, sin embargo, que Weber no menciona los afanes expansionistas del gobiemo norteamericano como un factor esencial para que México perdiera sus territorios norteños.

Los temas más debatidos y que mayor interés han despertado en ambos lados de la frontera, desde hace casi ciento cincuenta años, son los relacionados con la rebelión texana de 1836 y la guerra mexicano-norteamericana de 1846-1848. Las obvias consecuencias que estos conflictos trajeron a cada una de las historias nacionales los hacen un objeto de estudio que ha despertado frecuentemente sentimientos nacionales en los 
uutores, mismos que en muchas ocasiones desvirtúan el análisis histórico. Desafortunadamente, la mayor parte de la historiografía de los últimos veinte años años tiende todavía a repetir los mismos errores.

Entre los procesos que se dieron en la frontera entre México y Estados Unidos en la primera mitad del siglo XIX, destaca por su novedad e Importancia historiográfica el del comercio internacional. El comercio de Sunta Fe, historiado desde hace muchos afios, encontró a su bibliógrafo en Jack D. Ritten House con su The Santa Fe Trail: A Historical Bibliography (Albuquerque, Unversity of New Mexico, 1971) y nuevos trabajos monográficos, que algo agregan a lo ya conocido sobre el tema, con Britches: The Santa Fe Trail (College Station, Texas A\&M University, 1971), de Seymour V. Connor y Jimmy Skaggs y El comercio de Santa Fe y la guerra del ' 47 (México, Secretaría de Educación Pública, 1976) de Angela Moyano.

Los fenómenos que fueron preparando los acontecimientos bélicos de Texas, así como su influencia en las relaciones diplomáticas entre México y los Estados Unidos, han sido el campo de numerosísimos estudios. En las dos últimas décadas, la historia diplomática ha sacado a la luz y producido nuevas interpretaciones sobre el significado de las relaciones previas entre los dos países en el establecimiento de dos conflictos internacionales, siendo notable la tenacidad con que los investigadores mexicanos y norteamericanos defienden casi siempre las posturas oficiales de sus países. Los documentos de la relación de México con los Estados Unicios (México, UNAM, 1983-1985), preparados por Carlos Bosch y los libros Mexico Views Manifest Destiny, 1821-1846: An Essay on the Origins of the Mexican War (Albuquerque, University of New Mexico, 1975) de Gene Brack; y México-Estados Unidos; orígenes de una relación, 1819-1861 (México, Secretaría de Educación Pública, 1987) de Angela Moyano muestran la perspectiva mexicana, mientras que Too Far North... Too Far South (Los Angeles, Westernlore Press, 1967) de Odie B. Faulk; North American Divided: The Mexican War (New York, Oxford University, 1971) de Odie B. Faulk y Connor V. Seymour; The Diplomacy of Annexation, Texas, Oregon and the Mexican War (Columbia, University of Missouri, 1973) de David M. Pletcher y Mr. Polk's War; American Opposition and Dissent, (Madison, University of Wisconsin, 1973) de John $\mathrm{H}$. Schroeder lo hacen desde la norteamericana.

Si los asuntos diplomáticos que precedieron al conflicto armado de 1846-1848 son muy polémicos, tanto más será el tratar de la guerra en sí. Aunque existe una numerosísima bibliografía sobre este conflicto desde finales del siglo pasado, el tema de la guerra del ' 47 sigue esperando un análisis serio y objetivo, Los libros como The Mexican War, 1846-1848 
(New York, Macmillan, 1974) de K. Jack Bauer, La guerra del ' 47 y la resistencia popular a la ocupación (México, Nuestro Tiempo, 1976) de Gilberto López y Rivas; Blood over Texas, The Truth about Mexico's War with the United States (New York, Julian Messner, 1976) de Sanford H. Montaigne y La guerra del 47 y la opinión pública (México, Secretaría de Educación Pública, 1975) de Jesús Velasco Márquez poco agregan para el entendimiento del fenómeno. Más útiles son las antologías Mexicanos y norteamericanos ante la guerra del 47 (México, Secretaría de Educación Pública, de 1977) de Josefina Zoraida Vázquez y Chronicles of the Gringos; The U.S. Army in the Mexican War, 1846-1848: Accounts of Eyewilnesses (Albuquerque, University of New Mexico, 1968) de George W. Smith y Charles Judah, o las descripciones regionales de la guerra en Baja California como Testimonios de la invasión a Baja California, 1846-1848 (Tijuana, Universidad Autónoma de Baja California, 1984) de Angela Moyano y The Mexican War in Baja California (Los Angeles, Dawson's Book Shop, 1977) de Doyce B. Nunys.

Con la pérdida de más de la mitad del territorio mexicano y la posterior compra de La Mesilla, la frontera entre México y Estados Unidos adquirió su actual localización y las regiones al norte de ella se vieron inmiscuidas con los procesos históricos pertenecientes a los Estados Unidos, mientras que al sur de ella se consolidaba la soberanía mexicana. De ahi que la historia de la frontera adquiera finalmente la dualidad que le es inherente.

Las partes que se integraron a los Estados Unidos se vieron primeramente inmersos en lo que se conoce en la historia norteamericana como el "Movimiento al Oeste", cuyo estudio corresponde a la escuela llamada Western Americana. Esta corriente historiografica ha producido abundantes monografías sobre lo que es ahora el suroeste de los Estados Unidos sin darle mayor importancia a su situación fronteriza, centrándose en temáticas tales como biografías de "pioneros" norteamericanos y sus empresas, los progresos de la colonización angloamericanos, las guerras con los indios, la búsqueda de recursos naturales, etc. Por lo mismo, sus trabajos son casi siempre inútiles para los temas que nos ocupan.

La corriente de estudios regionales - 0 "U. S. Southwest History"en cambio, ofrece algunos trabajos sobre la estructura económica y social de la región norteamericana fronteriza con México así como de la población de origen mexicano que en ella habita.

El complicado proceso de la ratificación de las mercedes de tierras de origen novohispano y mexicano y su historia legal ha logrado interesar a un buen número de historiadores norteamericanos debido a la importacia económica local de esas grandes extensiones de terreno. En especial durante la última década, se ha llevado a cabo una enorme cantidad de 
estudios fuertemente monográficos, la mayor parte de ellos dedicados a una de estas concesiones de tierra y publicados en forma de artículos en revistas especializadas. Sobresalen por su visión más general y por la profundidad de su análisis The Court of Private Land Claims and the Adjudication of Spanish Land Grant Titles, 1891-1904 (Albuquerque, University of New Mexico, 1971) de Richard W. Bradfute; Land, Water and Culture: New Perspectives on Hispanic Land Grants (Albuquerque, University of New Mexico, 1987) de Charles L. Briggs y John R. Van Ness; Mercedes Reales; Land Grants on the Upper Rio Grande Region (Albuquerque, University of New Mexico, 1986) de Victor Wesrphall; y Spanish and Mexican Land Grants in the Chihuahua Acquisition (EI Paso, Texas Western Press, 1971) de J. J. Bowden.

De mayor interés es el desarrollo económico y social de esa extensa región entonces habitada moyoritariamente por personas de origen mexicano, la escuela historiográfica chicana es la que nos brinda, en este sentido, nuevas perspectivas e interpretaciones sobre la realidad social del suroeste norteamericano en la segunda mitad del siglo XIX. A pesar de contar con pocos años de vida, de 1965 al presente, la escuela chicana ha resultado ser una de las más prolíficas del momento. Sus estudios innovadores sobre su propia comunidad han abierto nuevos caminos a la investigación histórica en general y eso se ha reflejado en una enorme producción. Partiendo de estudios generales como los antes mencionados de Rudolfo Acuña, Mario Barrera, David Montejano, etc., los estudiosos chicanos han podido comenzar a recomponer su historia a partir del estudio de su conformación como minoría nacional a finales del siglo pasado. Han surgido entonces investigaciones sobre Ios chicanos en el siglo XIX en California con Chicanos in a Changing Society: From Mexican Pueblos to American Barrios in Santa Barbara and Southern California, 18481930 (Cambridge, Harvard University, 1974) de Albert Camarillo; The Los Angeles Barrio, 1850-1890: A Social History (Berkeley, University of California, 1979) de Richard Griswold del Castillo y The Decline of the Californios: A Social History of the Spanish-Speaking Californians, 18461890. (Berkeley, University of California, 1970) de Leonard Pitt. Acerca de Nuevo México en el siglo XIX se han escrito, entre otros trabajos: The Spanish Americans of New Mexico: A Heritage of Pride (Albuquerque, University of California, 1980) de Roxanne D. Ortiz; Mexicano Resistance in the Southwest: "The Sacred Right of Self-Preservation" (Austin, University of Texas, 1981) de Robert J. Rosenbaum y Political History of New Mexico, (s.p.i., 1971) de Daniel Valdez Tapia. Texas es, con mucho, el estado donde se ha estudiado más el proceso de formación del pueblo chicano. Entre la abultada producción historiográfica se encuentran: $A$ 
Social History of Mexican Americans in Nineteenth Century Duval Coun ty (San Diego, Duval County Commisioners, 1978), The Tejano Community, 1836-1900 (Albuquerque, University of New Mexico, 1982) y They Called Them Greasers: Anglo Attitudes toward Mexicans in Texas, 1821 1900 (Austin, University of Texas, 1983) de Genaro De León; Desserı Immigrants: The Mexicans at El Paso, 1880-1920 (New Haven, Yale University, 1981) de Mario T. García; Anglos and Mexicans in the Making of Texas, 1836-1986 (Austin, University of Texas, 1987) de David Montejano; Chicano Revolt in a Texas Town (Notre Dame, University of Notre Dame, 1974) de John S. Shockley; Anglo-Americans and Mexican-Americans in South Texas (New York, Arno, 1974) de Ozzie Simmons y El movimiento obrero mexicano en el sur de Texas 1900-1920 (México, Secretaría de Educación Pública, 1986) de Emilio Zamora.

Las regiones mexicanas que quedaron adyacentes a la línea fronteriza, que se trazó al finalizar la guerra contra Estados Unidos, se vieron afectadas al convertirse en provincias fronterizas. Esta situación influyó para que su integración a los grandes movimientos de la historia de México adquiera características especiales que lógicamente se reflejan en su escasa historiografía, misma que todavía falta mucho en desarrollarse.

Sobre las incursiones de los filibusteros en la frontera noroccidental de México, por ejemplo, un tema al cual se le habían dedicado varias monografías en la primera mitad del siglo, sólo encontramos que para los últimos veinte años se publicaron: Agents of Manifest Destiny: The Lives and Times of Filibusters (Chapell Hill, University of North Carolina, 1979) de Charles F. Brown; ; Y Caborca se cubrió de gloria...! La expedición filibustera de Henry Crabb a Sonora (México, Porrúa, 1976) de J. Antonio Ruibal Corella y The Republic of Lower California, 1853-1854 (Los Angeles, Dawson's Book Shop, 1968) de Arthur Woodward.

Las vicisitudes políticas propias de los estados fronterizos al sur de la línea fronteriza y su articulación con la historia nacional de México ha sido un campo también abandonado por completo por los investigadores en las últimas dos décadas. Las historias colectivas estatales Panorama histórico de la Baja California (Tijuana, Instituto de Investigaciones Históricas UNAM-UABC, 1983) e Historia general de Sonora (Hermosillo, Gobierno del Estado, 1985) son de las pocas que contienen pormenorizadas relaciones sobre temas políticos locales del siglo XIX, además de presentar buenas síntesis sobre las historias de esos estados, La tradición de reseffar el efecto o desarrollo de los grandes movimientos nacionales en escalas locales ya no es tan común en las últimas décadas, aunque se han dado a conocer $\mathrm{La}$ Intervencion y el Imperio en el estado de Chihuahua (Chihuahua, Litografía REGMA, 1972) de Francisco R. Almada; La carrera pública de Don 
Ramón Corral (México, Secretaría de Educación Pública, 1976) de Jesús Luna y Manuel Z. Gómez, gobernador y comandante de Nuevo Leon durante la Intervención Francesa (Monterrey, Ed. Alfonso Reyes, 1977) de Rodolfo Arroyo Llano.

Otros estudios, escasos también, presentan la novedad de intentar conjuntar los avatares políticos con las estructuras económicas y sociales locales. Tales son: Sonora Strongman, Ignacio Pesqueira and His Times (Tucson, University of Arizona, 1974) de Rudolfo Acuna; Economla de guerra y poder regional en el siglo XIX, gastos militares, aduanas y comerciantes en los años de Vidaurri (1855-1864) (Monterrey, AGENL, 1983) de Mario Cerruti. Siguiendo esta temática particular, On the Periphery of Nineteenth Century Mexico, Sonora and Sinaloa, 1810-1877 (Tucson, Unversity of Arizona, 1982), de Stuart F. Voss nos presenta un agudo análisis de los grupos de poder en estos dos estados y las estructuras económicas que los apoyan.

La investigación sobre temas económicos, especialmente en el desarrollo de la agricultura capitalista y la industrialización, se encuentra todavía en sus etapas iniciales, pero se empiezan a dar a conocer ya varios de sus frutos como Abriendo tierras en Mexicali (Tijuana, Instituto de Investigaciones Históricas UNAM-UABC) de Irma Apodaca; Burguesía y capitalismo en Monterrey (México, Claves Latinoamericanas, 1983) y "División capitalista de la producción, industrias y mercado interior. Un estudio regional: Monterrey (1890-1910)" en El siglo XIX en México; Cincu procesos regionales: Morelos, Yucatán, Jalisco y Puebla (México, Claves Latinoamericanas, 1985) de Mario Cerruti; Samuel Peter Heintzelman and the Sonora Exploring Mine Co. (Tucson, University of Arizona, 1980) de Diane M. T. North; Colonel Green and the Copper Skyrocket, (Tucson, University of Arizona, 1976) de C. L. Sonnichsen; y Losorlgenes de la industrialización en Monterrey. Una historia económica y social desde la calda del Segundo Imperio hasta el fin de la Revolución (18671920) (Monterrey, ITESM, 1969) de Isidro Vizcaya Canales.

\section{c) Siglo $x x$}

A partir de 1910, con el estallido de la revolución mexicana, el papel de la frontera en la historia del país cambió completamente. De ser un lugar alejado e incógnito de México se convirtió en una región que invadió el centro del país, en una "frontera nómada", como la llamó Héctor Aguilar Camín, que se extendió para dominar la vida política nacional desde los tiempos de la huelga de Cananea hasta el final del maximato de Plutarco Elías Calles. Asimismo, la frontera mexicano-norteamericana se conmo- 
vió con el movimiento armado y generó algunos de los fenómenos que liı caracterizan hasta el día de hoy y las relaciones diplomáticas entre los dos países nunca volvieron a ser iguales. La frontera, durante la década violenta de la revolución, significó para los mexicanos el campo de muchas batallas decisivas, un refugio para los exiliados, lugar de intriga y conspiraciones, puerto del comercio ilegal de armas, punto de salida de los civiles que huían de la guerra $y$, en ocasiones, la oportunidad de crear conflictos internacionales.

La bibliografía reciente sobre la revolución mexicana es, por supuesto, enorme. Dentro de ella, los estudios que se refieren a su desarrollo e influencia en las zonas fronterizas son también muy numerosos debido a la gran riqueza de la temática revolucionaria. Esto ha motivado que surjan varios trabajos de sintesis sobre la revolución en la frontera mexicananorteamericana.Fragments of the Mexican Revolution:Personal Accounts from the Border (Albuquerque, University of New Mexico, 1969) de Oscar J. Martínez, es una cuidadosa recopilación de testimonios que muestra, ante todo, la riqueza de las perspectivas con que se contempla un hecho histórico tan rico en manifestaciones. Revolution on the Border: The U.S. and Mexico (Albuquerque, University of New Mexico, 1988) de Don Coerver y Linda B. Hall, en cambio, es un excelente trabajo de investigación que conjuga los diversos temas de la Revolución en la frontera para presentarlos como un conjunto coherente y comprensible de los hechos. Para un enfoque más específico sobre las finanzas fronterizas, se puede consultar a Michael D. Carman, United States Customs and the Madero Revolution (El Paso, Texan Western Press, 1976).

Algunos de los diversos problemas que generó la revolución Mexicana en toda la franja fronteriza del norte han sido investigados, pero generalmente de manera aislada. La vida y actividades de los exiliados políticos, por ejemplo, ha sido el objeto de estudio de: Revoltosos: Mexico's Rebels in the United States, 1903-1923 (College Station, Texas A\&M University, 1981) de W. Dirk Raat y Mexican Exiles in the Borderlands, 1910-1913 (El Paso, University of Texas, 1979) de Peter V. M. Henderson. Otro aspecto, el de la seguridad nacional de la región fronteriza norteamericana durante la crisis armada, ha sido examinado en Blood on the Border: The United States Army and the Mexican Irregulars (New York, Macmillan, 1969) de Clarence C. Clendenen; Wings over the Border: The Army Air Service Armed Patrol of the United States-Mexican Border, 1919-1921 (E1 Paso, Texas Western Press, 1970); de Stancy C. Hinkle; The Border Wardens (Englewood Cliffs, Prentice Hall, 1971) de John Myers y Border Patrol: With the U.S. Immigration Service on the Mexican Boundary, 1910-1954 (El Paso, Texas Western Press, 1978) de Clifford A. Perkins. 
Las relaciones diplomáticas que se dieron en esta época entre los dos países han merecido un buen número de estudios académicos, algunos de los cuales reseñan ciertos problemas que se originaron en la frontera y trataron de ser resueltos por las autoridades oficiales de México y Estados Unidos. Entre ellos se encuentran: La revolución mexicana de 1910-1917 y la politica de Estados Unidos (México, Cultura Popular, 1976) de M. S. Alperivich y B. T. Rudenko; Diplomacy and Revolution: United StatesMexican Relations under Wilson and Carranza (Tucson, University of Arizona, 1971) de Mark T. Gilderhus; The United States and Huerta (Lincoln, University of Nebraska, 1969) de Kenneth J. Grieb; Revolution and Intervention: The Diplomacy of Taft and Wilson with Mexico, 19101917 (Cambridge, MIT, 1970) de Edward P. Haley: The Secret War in Mexico; Europe, The United States and the Mexican Revolution (Chicago, University of Chicago, 1981) de Friedrich Katz; Luther E. Ellsworth: $U$. $S$. Consul on the Border during the Mexican Revolution (El Paso, Texas Western Press, 1975) de Dorothy P. Kering; William Howard Taft and the United States Foreign Policy: The Apprenticeship Years, 1900-1918, (Chicago, University of Illinois, 1975) de Ralph E. Minger y La Revolution intervenida; Relaciones diplomáticas entre México y Estados Unidos (1910-1914) (México, El Colegio de México, 1971) de Berta Ulloa.

Dentro de los trabajos más específicos, un primer grupo de monografías se podría formar con aquellas obras que se refieren a los acontecimientos locales y estatales durante los años revolucionarios. En Baja California, el conflicto que ha causado un mayor interés en los investigadores desde siempre ha sido el del movimiento magonista de 1911, El trabajo de Juan Gómez- Quiñones, Sembradores: Ricardo Flores Magón y el Partido Liberal Mexicano; A Eulogy and Critique (Los Angeles, Chicano Studies Center, UCLA, 1973) revivió viejas polémicas sobre la naturaleza y propósitos del Plan de San Diego, de manera que varios estudiosos del tema, como Charles C. Cumberland, Allan Gerlach, Willian Hager, Louis R. Sadler, James A. Sandos y el propio Gómez-Quifiones se vieron obligados a publicar varios artículos en defensa de sus posturas.

El estado de Sonora cuenta con el libro ya clásico de Héctor Aguilar Camín La frontera nómada: Sonora y la revolución mexicana (México, Siglo XXI, 1977), en donde el autor describe con una ágil prosa y una gran profundidad analítica las condiciones sociales que hicieron posible el surgimiento del grupo revolucionario sonorense, el que a la postre sería el verdadero triunfador, y los primeros pasos de éste dentro de la rebelión armada. Una narración más vivida y subjetiva, escrita por un participante es La revolución en Sonora (Hermosillo, Gobierno del Estado, 1969) de Antonio G. Rivera. Dos bibliografías de gran calidad acerca de protago- 
nistas de la revolución sonorense, ambos finalmente derrotados son José Maria Maytorena and the Revolution in Sonora 1910-1915 (tesis doctoral, Lincoln, University of Nebraska, 1974) de Susan Mc Clymont Deeds y Emilio Kosterlitzky: Eagle of Sonora and the Southwest Border (Glendale, Arthur H. Clark, 1970) de Cornelius C. Smith.

Una de las regiones de mayor importacia dentro del desarrollo de la revolución fue Chihuahua, el feudo de Pancho Villa y escenario de sus constantes problemas con el gobierno y las autoridades norteamericanas que le brindaban su apoyo. La fuerza de este personaje tal vez ha predominado tanto en el ámbito chihuahuense, que pocos son los estudios que se refieren a la actividad revolucionaria de otros caudillos en ese estado. Existen, sin embargo, dos excelentes análisis sobre otros personajes locales de importancia: Insurgent Governor: Abraham Gonzalez and the Mexican Revolution in Chihuahua ( Lincoln, University of Nebraska, 1973) de William Beesley y Mexican Rebel: Pascual Orozco and the Mexican Revolution, 1910-1915 (Lincoln, University of Nebraska, 1967) de Michael Meyer.

La enorme frontera de Texas con cuatro estados mexicanos fue desde el comienzo de la revolución maderista un importante lugar de asilo político. Los estudios sobre la revolución en estos estados son, sin embargo, más bien escasos. Los libros de Don M. Coerver y Linda B. Hall, Revolution on the Rio Grande: Governor Colquitt of Texas and the Mexican Revolution, 1911-1915 (San Antonio, Trinity Border Institute, 1981) y Texas and the Mexican Revolution: A Study in State and National Border Policy, 1910-1920 (San Antonio, Trinity University, 1984) analizan detalladamente las relaciones entre las diversas oficinas gubernamentales de los dos países mostrándonos en casos concretos la aplicación de las doctrinas diplomáticas sostenidas por México y Estados Unidos. Acerca de la región al sur del río Bravo, encontramos sólo a: La revolución mexicana en Coahuila, etapa maderista (Saltillo, AEPS, 1968) de José de la Luz Valdés; La revolución mexicana en el estado de Tamaulipas. Cronologla (México, Manuel Porrúa, 1973-1976) de Ciro de la Garza Trevifo e Historia de la revolución mexicana en Coahuila (México, Talleres Gráficos de la Nación, 1970) de Ildefonso Villarelo.

La misma revolución provocó el inicio de uno de los fenómenos más característicos de la frontera mexicano-norteamericana en el presente siglo: la emigración masiva de trabajadores de México a los Estados Unidos. Este proceso constante de transferencia de recursos humanos llamó la atención de los estudiosos desde su mismo comienzo. Por ello, los primeros trabajos académicos sobre el tema, de carácter más bien sociológico y antropológico que historiográfico, datan de las décadas de 
los veinte y los treinta, sobresaliendo entre ellos los libros de Manuel Gamio y Paul S. Taylor. Con el paso del tiempo, los historiadores se fueron interesando poco a poco en este fenómeno, de forma que para mediados de la década de los sesenta, cuando se consideró que estaba ya dada la posibilidad de estudiar este problema bajo una perspectiva histórica, se comenzó a producir una robustísima literatura sobre el tema. La extraordinaria producción de trabajos sobre la migración mexicana a Estados Unidos contempla prácticamente todos los aspectos por los cuales puede ser analizado este tema de estudio. Los libros nombrados a continuación son sólo un pequeñísimo fragmento de la producción total de los últimos veinte afoos sobre esta temática: Migration Across Borders: Mexico and the United States (Albany, New York State University, 1979) de Fernando Cámara y Ruben Van Kemper; "United States Immigration Policies toward Mexicans: An Historical Perspective" en: Chicago Law Review (vol. II, verano de 1975), de Gilberto Cárdenas; Mexican Emigration to the United States, 1897-1931: Socioeconomic Patterns (Tucson, University of Arizona, 1980) de Lawrence A. Cardoso; Los mexicanos que devolvi6 la crisis, 1929-1932 (México, Secretaría de Relaciones Exteriores, 1974) de Mercedes Carreras de Velasco; The Development of the Migratory Farm Labor System in Texas, 1900-1954 (San Francisco, R\&E Research Associates, 1977) de George O. Coalson; The Bracero Program: Interest Groups and Foreing Policy (Austin, University of Texas, 1971) de Richard B. Craig; Farm Workers and Agri Bussiness in California, 1947-1960 (Notre Dame, Unversity of Notre Dame, 1977) de Emesto Galarza; Unwanted Mexican Americans in the Great Depression: Repatriation Pressures, 1929-1939 (Tucson, University of Arizona, 1977) de Abraham Hoffman; Anglo over Bracero: A history of the Mexican Worker in the United States from Roosevelt to Nixon (San Francisco, R\&E Research Associates, 1977) de Peter N. Kistein; American Attitudes toward Mexican Immigration, 1924-1956 (San Francisco, R\&E Research Associates, 1971) de Robert J. Lipshultz; Mexican Immigration to the United States, 19101930 (San Francisco, R\&E Research Associates, 1971) de Juan R. Martínez.

La escuela historiográfica chicana ha seguido estudiando los factores que han afectado su desarrollo en este siglo. Debido a su expansión geográfica por los Estados Unidos, ya no toda la historia chicana tiene relación directa con la historia de la frontera mexicano-norteamericana. Sin embargo, la gran mayoría de sus trabajos sí se refieren a los habitantes de las zonas fronterizas. Algunos de los mejores estudios chicanos para el siglo XX son: Los Chicanos, una minorla racial explotada (México, Nuestro Tiempo, 1971) de Gilberto López y Rivas; Al norte del rio Bravo, pasado cercano, 1930-1980 (México, Siglo XXI, 1983) de David R Ma-. 
ciel; Aztlán; Historia contemporánea del pueblo chicano, 1910-1972 (México, Secretaría de Educación Pública, 1976) de David R. Maciel y Patricia Bueno, Tijerina and the Courthouse Raid (Albuquerque, University of New Mexico, 1969) de Peter Nabokov; Los mexicanos de los Estados Unidos y el Movimiento Chicano (México, Fondo de Cultura Económica, 1972) de Joan W. Moore; Chicano, The Evolution of a People (Minneapolis, Wiston, 1973) de Renato Rosaldo, et al.; Chávez: la interminable batalla por la dignidad (México, Editores Asociados Mexicanos, 1979) y Chávez and the Farm Workers (Boston Beacon, 1975) de Ronald Taylor; y Chicano Elites and Non-Elites in South Texas (Palo Alto, R\&E Research Associates, 1979) de Roberto Villarreal.

Los acontecimientos más cercanos a nuestros días, como serían el incremento del comercio internacional, el desarrollo económico general de las regiones fronterizas de México y Estados Unidos, las políticas oficiales para impulsar ese desarrollo, el contrabando, la utilización de las industrias maquiladoras, los flujos de capital, el progreso industrial y agrícola, los problemas ecológicos, etcétera, son abordados de forma casi exclusiva por diversos científicos sociales entre los que se encuentran historiadores. La tendencia actual a integrar equipos interdisciplinarios de investigación aún no es práctica común entre los profesionales de la historia. Debido a esto, entre la abrumadora biliografía que de estos temas existe, pocos trabajos sobre el desarrollo comtemporáneo de la frontera se pueden considerar estrictamente historiográficos, y éstos se reducen a unos pocos temas. El movimiento chicano de mediados dé los sesenta logró atraer la atención casi inmediata de varios investigadores, pero la naturaleza de su trabajo es más bien de índole periodística.

Acerca de temas económicos, se han escrito obras de cierto contenido historico tan diverso como: The Border Industrialization Program of Mexico (Lexington, Hearth, 1971) de Donal W. Baerrensen; La modernización de la agricultura mexicana, 1940-1970 (México, Siglo XXI, 1982) de Cynthia Hewitt; Agrarian Populism and the Mexican State. The Struggle for Land in Sonora (Berkeley, University of California, 1981) de Steven E. Sanderson; y Nacimiento y fracaso del algodon, Matamoros (1938-1965) (Reynosa, Instituto de Investigaciones Históricas, UAT, 1980) de Teodoro Hernández Acosta.

Podemos afirmar que los aspectos y manifestaciones culturales de la región fronteriza han sido frecuentemente desdeñados por los investigadores. Este tipo de estudios son publicados por lo general en revistas regionales de muy escaso tiraje, y han sido los escritores y los críticos literarios quienes más se han avocado al análisis e interpretación de la cultura fronteriza. Por lo demás, otra característica de los estudios cultu- 
rules es su especificidad, pues casi no existen ensayos que ofrezcan un panorama cultural extenso. Una excepción es el brillante prólogo de Carlos Monsiváis "De México y los Chicanos; México y su cultura fronteriza" publicado en La otra cara de México; el pueblo chicano (México, El Caballito, 1977). En este ensayo, Monsiváis desarrolla una aguda interprelación de la visión de la frontera que predomina en el centro de México y determina los temas fundamentales de la cultura fronteriza. Un libro que ha incluido varios ensayos importantes sobre la cultura es Aztlán y México (New York, Bilingual Press) del destacado crítico Luis Leal. A través de la frontera (México, CESTEM, 1982) también incluye importantes ensayos que tratan de la experiencia cultural fronteriza en los que se tratan principalmente las diversas manifestaciones de la cultural chicana, especialmente las letras y el arte.

Quizá el aspecto cultural que más atención ha recibido por parte de los investigadores es la literatura. Se han publicado recientemente varias antologías de obras literarias, y muy pocos estudios críticos. La colección Frontera de la Secretaría de Educación Pública ha hecho un aporte sustantivo en este sentido al publicar los siguientes títulos: Primer encuentro de poetas y narradores jóvenes de la frontera norte (1986), Primer foro de cultura contemporánea del norte de México (1987), Tercer encuentro de poesía joven de la frontera norte (1987) y La literatura chicana (1988) de Charles M. Tatum. Gracias a estos estudios pioneros se está dando a conocer la larga y dinámica experiencia literaria de la frontera mexicanoestadounidense, esperiencia que no sólo refleja la esencia misma del ser fronterizo, sino que incluye los más variados géneros: narrativa, poesía, teatro, ensayo y crónica.

Al norte del Bravo los chicanos han desarrollado una cultura propia que cuenta con gran cantidad de matices. Las antologías y selecciones de ensayos son muy numerosas, pero sobresalen por su calidad Aztecas del norte (Greenview, Fawcett, 1973) compilado por Jack D. Forbes y Aztlán: An Anthology of Mexican American Literature (New York, Vintage Press s. a.) de Luis Valdez y Stan Steiner. Ambas antologías contienen valiosas introducciones y comentarios de los compiladores que tratan los diversos temas de la cultura fronteriza.

Por otro lado, la frontera México-Estados Unidos ha sido uno de los temas más recurrentes de la cinematografía de ambos países. Desde las primeras producciones norteamericanas la frontera fue una temática utilizada por el cine de la década de los treinta. En un sinnúmero de "westerns", comedias y películas de tema policiaco, la frontera era el sitio donde se desarrollaba la acción. A través de ellos se fue creando y consolidando un estereotipo de la frontera. El destacado historiador y crítico del cine 
mexicano, Emiliano García Riera, analiza el significado de estas producciones en su ambicioso estudio México visto por el cine extranjero (México, ERA, 1987-1989, 6 vols.). Esta obra es el resultado de una labor de investigación impresionante y seguramente será el punto de partida de nuevos estudios sobre el tema.

La obra cinematográfica mexicana sobre la frontera es estudiada por Norma Iglesias en su trabajo Visión de la frontera en el cine mexicano (Tijuana, Colegio de la Frontera Norte de México, 1987). En este ensayo la autora desarrolla una periodización del cine fronterizo mexicano desde la década de los veinte hasta la de los setenta. Es un primer intento para acercarse al estudio del cine fronterizo, su única limitación es su brevedad. Un segundo trabajo panorámico sobre estos temas son los breves artículos de Emilio García Riera que aparecieron en el diario La Opinión de Los Angeles en enero de 1984. En ellos, se discuten tanto el cine fronterizo como el norteamericano.

Diferente en propósito y periodo analizado es el capítulo "La frontera grifa" del libro La condición del cine mexicano (México, Posada, 1986) escrito por el conocido crítico de cine Jorge Ayala Blanco. Con aguda sensibilidad y conocimiento del tema analiza aquí el cine de la frontera en los últimos dos sexenios. Este estudio es indispensable para la comprensión del cine fronterizo mexicano.

El único estudio cinematográfico que incluye las resefias de producciones es Chicano Cinema, compilado por Gary D. Keller (New York, Bilingual Review Press, 1985). No sólo es el trabajo más amplio sobre el florecimiento del cine chicano, sino que muchos de sus ensayos y reseñas son importantes para el estudio de la frontera por sí misma. Algunos de los trabajos que se incluyen en este libro son: "Chicano in Film; History of an Image" de Carlos Cortés y "Visions of the Other Mexico; Chicanos and Undocumented Workers in Mexican Cinema, 1954-1982" de David R. Maciel. Recientemente la Cineteca Nacional tradujo al español y publicó este libro.

Las manifestaciones artísticas de la frontera no han sido estudiadas ampliamente, ni como arte regional ni en sus aspectos individuales de las artes en los dos países. Al norte de la frontera, el florecimiento de las artes plásticas y el muralismo chicano son los temas que han merecido más estudios académicos. Los artistas fronterizos, en general, han empezado a recibir el reconocimiento del público y la atención de los investigadores apenas en los últimos años. Uno de los libros más documentados sobre el tema es Mexican-American Artists (Austin, University of Texas Press, 1973) de Jacinto Quirarte. A través de una sensata periodización del desarrolloartístico chicano desde la Colonia hasta principios de los setenta, 
1) uutor demuestra claramente la rica tradición del arte chicano en sus más destacadas manifestaciones artísticas. Este estudio refleja tambien las influencias predominantes del arte mexicano en el arte chicano sin menospreciar la originalidad y el carácter dinámico del arte chicano. El muralismo y la pintura son los géneros más estudiados en Mexican American Artists.

Un estudio más reciente y panorámico es Arte Chicano: A Comprehensive Annotated Bibliography of Chicano Art, 1965-1981 (Berkeley, Chicuno Studies Library Publications Unit, University of California) compilado por Shifea M. Goldman y Tomás Ybarra- Frausto. Aparte de ser la bibliografía comentada más extensa sobre el tema, este libro contiene un importante ensayo introductorio que ofrece también una periodización de lus manifestaciones artísticas desde tiempos coloniales hasta $1980 . \mathrm{La}$ conclusión apunta las corrientes y las características principales de los movimientos artísticos chicanos.

\section{IIACIA UN BALANCE}

En los últimos veinte años, la producción sobre la frontera México-Esuddos Unidos ha sido impresionante y voluminosa, tanto en cantidad como on calidad. Muchos investigadores de ambos países, europeos e incluso wiático han publicado ensayos y monografías sobre estos temas.

En términos cuantitativos, las publicaciones acerca de los aspectos politicos y económicos son las que predominan en virtud de su importancia Inmediata y vital tanto para México como para Estados Unidos. Asimismo, ol icma de la emigración mexicana y latinoamericana hacia Estados Unidos on sus diversas manifestaciones han sido objeto de una amplia investigaulon. Finalmente, las cuestiones ecológicas y las relacionadas con los recursos naturales de la región fronteriza, así como el tema del narcotráfi00, han sido el centro de atención de numerosos estudios académicos en low últimos años. Es conveniente hacer notar que esta impresionante producción se ha desarrollado en el ámbito de las ciencias sociales.

Desde una perspectiva historiográfica, existen ciertos periodos y temas nobre los que se han publicado un número mayor de estudios de la colonia, In revolución de 1910 y la época posterior a la Segunda Guerra Mundial, en tunto que la segunda mitad del siglo XIX y el periodo comprendido entre 1020 y 1940 han recibido una menor consideración por parte de los investigadores. Las historias de las instituciones, diplomática, urbana y laboral de la frontera son las que han predominado en términos generales, mientras que las historias social, bibliográfica y cultural aún esperan a los lihuriadores que las cultiven. 
Aunque es indudable que, al paso de los affos, se han podido realizar innumerables investigaciones, también es cierto que este progreso plantea nuevos problemas e insinúa la continuación de otros trabajos. Se puede prever con facilidad que las futuras investigaciones historicas sobre la frontera tenderán a integrarse a las demás ciencias sociales, tal cual éstas lo están haciendo en diversos programas de desarrollo interdisciplinario. Así, se espera una mayor producción de obras de historia social y económica de la frontera entre México y Estados Unidos en varios periodos.

El siglo XIX es ahora la época más abandonada por los investigadores, pero es de esperarse que nuevas técnicas y supuestos teóricos motiven la realización de estudios en áreas que hasta ahora no han sido analizadas. Algunos personajes importantes para la vida política, como Juan N. Cortina o Juan N. Seguín, requieren de bibliografias serias y objetivas. Hacen falta asimismo estudios comparativos sobre ciudades, regiones o estados que se encuentren en ambos lados de la frontera. Un estudio histórico de esta clase enriquecería enormemente las perspectivas de investigación. Historias laborales y culturales son ahora necesarias para poder marcar una línea de evolución de las economías agrícolas del siglo pasado a las industrias del presente.

Acerca del siglo $\mathrm{XX}$, es imprescindible comenzar la exploración seria del tema de investigación más importante de los próximos años: la historia económica de ambas regiones fronterizas a partir de 1940. Otros temas, como la evolución de las culturas populares o los estudios familiares y de la mujer, se deberán ir desarrollando a la par que las demás ciencias sociales aporten nuevos métodos a la historia. Es posible que la historia comparada pueda ofrecer una novedosa perspectiva de análisis que hasta ahora no se ha empleado para el estudio de la frontera México-Estados Unidos. Relacionada con este enfoque está la posibilidad de integrar equipos de trabajo con investigadores de ambos países. Queda, pues, a los estudiosos de la frontera mexicano-norteamericana un amplísimo campo por desarrollar mientras que se cuenta con el apoyo cada vez mayor de nuevas investigaciones. Se espera, entonces, que en los proximos affos la frontera entre México y Estados Unidos, como tema de estudio, mantenga su vitalidad, supere las lagunas de información todavía existentes y, sobre todo alcance el reconocimiento que se merece. 